\title{
Placenta pathology evaluation: are CAP guidelines useful? A preliminary analysis
}

\author{
Jennifer Steines, MD, ${ }^{1}$ Adebukola Ogundoyin, BS, ${ }^{2}$ Colleen Kennedy Stockdale, MD, MS ${ }^{1}$
}

Keywords: College of American Pathologists; guideline adherence; placenta, pathology; pregnancy

\section{INTRODUCTION}

The College of American Pathologists (CAP) established a revised set of recommendations for placental evaluation in 1997. In review of the literature and anecdotally, there is discrepancy regarding placental submission to pathology. We evaluated adherence to CAP guidelines for placental pathology and clinical utility of the results.

${ }^{1}$ Department of Obstetrics and Gynecology, Carver College of Medicine, University of lowa Hospitals and Clinics, lowa City, IA, 52242

${ }^{2}$ Carver College of Medicine, University of lowa Hospitals and Clinics, lowa City, IA, 52242

\section{METHODS}

Retrospective case review of deliveries performed at the University of lowa from January 1 through March 1, 2005. Results were categorized as reviewed (met CAP guidelines and sent to pathology), not reviewed (met CAP guidelines and not sent to pathology), and not indicated (did not meet CAP guidelines and sent to pathology).

\section{RESULTS}

Of 251 deliveries, 113 placentas were evaluated pathologically. 56 met criteria for evaluation and were sent to pathology. 54 met criteria and were not sent to pathology. Three did not meet criteria and were evaluated. PPROM $(25 \%)$ and preeclampsia (19.6\%) were the two highest clinical indications in which placentas were sent for evaluation. Pathological costs for reviewed group of placentas were $\$ 32,406$. Projected costs for those placentas not reviewed but meeting CAP guidelines were $\$ 34,567.77$ deliveries were performed via cesarean section. Cesarean deliveries $(n=36[63 \%])$ were evaluated more frequently than vaginal deliveries $(n=18[32 \%])$ when CAP guidelines were met. Vaginal deliveries ( $n=27[52 \%])$ were less likely to be evaluated as compared to cesarean deliveries $(n=13[25 \%])$ when CAP guidelines were

Please cite this paper as: Steines J, Ogundoyin A, Stockdale CK. Placenta pathology evaluation: are CAP guidelines useful? A preliminary analysis. Proc Obstet Gynecol. 2012 ;2(3):Article 5 [ 2 p.]. Available from: http://ir.uiowa.edu/pog/. Free full text article.

Corresponding author: Jennifer Steines, Department of Obstetrics and Gynecology, University of lowa, 51244 PFP, 200 Hawkins Drive, lowa City, IA 42242. jennifer-steines@uiowa.edu. 
met. Only one placenta pathology result (IUFD) was documented at the postpartum visit. There was no followup documentation identified for the remaining placentas evaluated pathologically.

\section{CONCLUSIONS}

It appears that providers do not follow a uniform set of criteria when submitting placentas for evaluation. Providers are more-likely to send placentas following cesarean section despite meeting CAP indication. There appears to be minimal clinical utility from pathological evaluation given lack of postpartum documentation. 